\title{
A Novel Dual-Band Retro-directive Reflector Array on Paper Utilizing Substrate Integrated Waveguide (SIW) and Inkjet Printing Technologies for Chipless RFID Tag and Sensor Applications
}

\author{
Sangkil Kim ${ }^{1}$, Benjamin Cook ${ }^{1}$, James Cooper ${ }^{1}$, Anya Traille ${ }^{2}$, Apostolos Georgiadis ${ }^{3}$, Hervé Aubert ${ }^{2}$ and \\ Manos M. Tentzeris ${ }^{1}$ \\ ${ }^{1}$ Georgia Institue of Technology, City, GA, 30305, USA \\ ${ }^{2} \mathrm{CNRS}$, LAAS, Toulouse, France \\ ${ }^{3}$ Centre Tecnologic de Telecomunicacions de Catalunya (CTTC), Catalunya, Spain
}

\begin{abstract}
In this paper, we propose the first dual-band retrodirective reflector array using Substrate Integrated Waveguide (SIW) and inkjet-printed technologies on flexible low-cost substrates, such as paper, for operability around 3.6GHz and 5.8GHz. In addition, it offers the versatility of multiband retro-directive designs potentially covering numerous RFID interrogation, sensing and communication bands, while it does not suffer from typical undesirable EM interference effects from commonly used microstrip and CPW feed-lines. The retrodirective properties of the proposed architecture have been verified through the fabrication and characterization of a benchmarking prototype.

Index Terms - Retro-directive array, substrate integrated waveguide, inkjet printing, flexible electronics, microwave antenna arrays, sensors.
\end{abstract}

\section{INTRODUCTION}

Retro-directive array technology has found many applications in military and communications systems, including collision avoidance sensors, RFID and solar power satellite systems [1]. In contrast to conventional smart antenna systems, retro-directive systems are capable of self-steering, transmitting an incoming wave back towards the direction of the transmitter, without requiring complicated signal processing in order to compute the angle of arrival of the incoming wave. As a result, features such as low complexity and low cost have led to their consideration in applications where factors such as cost and power dissipation are particularly important.

Recently, chipless RFID and sensors are receiving significant scientific and commercial interest due to the use of purely passive circuitry, and consequently their low cost [2]. Retro-directive technology has already been considered for RFID applications [3] and, in its simplest form, the Van Atta architecture presents itself as a candidate for chipless RFID and sensing systems. In passive antenna sensor circuits the radar cross section or back-scattered signal from the sensor varies depending on a desired sensing parameter. The performance of such systems however, is typically sensitive to the relative orientation between the interrogator and the sensor. Retro-directive systems due to their self-steering capability present an excellent candidate for passive antenna sensors [4] and RFIDs [5] with robust performance under a wide range of reader-sensor orientation scenarios[6].

Furthermore, substrate integrated waveguide (SIW) technology is an attractive technology for low cost, compact and high performance circuits due to ease of fabrication, low loss, and increased isolation compared to microstrip technology [7].

In this paper, a novel dual-band completely passive retrodirective array on paper substrate combining SIW and inkjet printing technologies is presented for the first time with numerous potential applications in chipless RFID tag and sensor applications. The proposed compact, dual band structure offers an increased potential for sensing functionality at two different frequencies and it does not suffer from undesired feed EM radiation at the junction discontinuities occurring when using microstrip or coplanar waveguide structures [8][9]. Furthermore, the design can be easily scaled up to millimeter wave frequencies offering additional advantages for compact circuit topologies and larger array size. A prototype is fabricated operating at $3.6 \mathrm{GHz}$ and $5.8 \mathrm{GHz}$ and its simulated performance is compared to measurements.

\section{SIW ANTENNA DESIGN}

The benchmarking paper-based slotted waveguide SIW antenna elements have been designed at the desired frequencies of $3.6 \mathrm{GHz}$ and $5.8 \mathrm{GHz}$. The geometry of the antenna is shown in Fig. 1. The antenna for 3.6 GHz (Fig 1(a)) consists of an SIW structure with two resonant slots while the antenna for $5.8 \mathrm{GHz}$ (Fig 1(b)) has four resonant slots. This number of slots per array was necessary because the total physical length of the antenna arrays should be comparable in order to interweave them to make a retro-directive Van Atta array. The length of the slots is chosen to resonate at the operating frequency of the antenna, and the slot spacing $\left(D_{\text {slot1 }}, D_{\text {slot2 }}\right)$ is approximately half guided wavelength in order to obtain maximum radiation in the broadside direction. The end section of the SIW is short-circuited so that it is necessary to keep the distance between the end section and the last slot in odd number of quarter wavelength at operation frequency. 


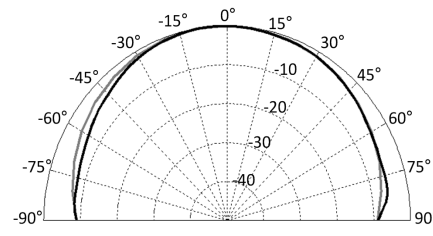

E-plane

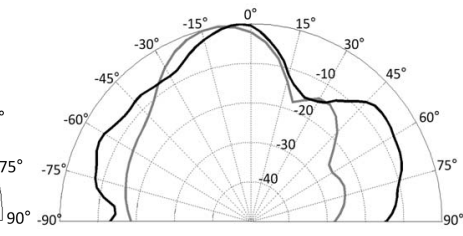

H-plane

(a)

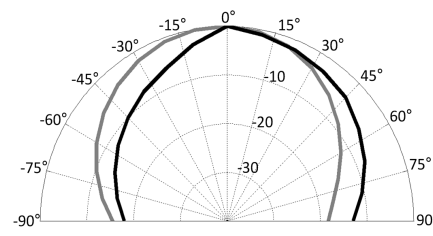

E-plane

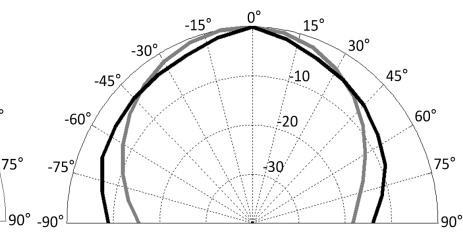

H-plane (b)

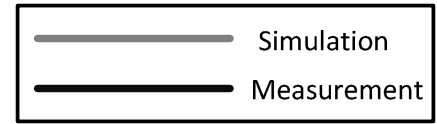

Fig. 3. Measured radiation patterns of the antennas for (a) $3.6 \mathrm{GHz}$ (b) $5.8 \mathrm{GHz}$

The length and the width of slots for $3.6 \mathrm{GHz}$ antenna array are $24 \mathrm{~mm}$ and $3 \mathrm{~mm}$ while those of slots for $5.8 \mathrm{GHz}$ are $17 \mathrm{~mm}$ and $1.15 \mathrm{~mm}$, respectively. The width of each antenna is chosen to keep the cut-off frequency of SIW lower than the operation frequency and the pitch of the vias is $1.6 \mathrm{~mm}$ which is two times larger than the diameter of a via. The simulated and measured reflection coefficients $\left(\mathrm{S}_{11}\right)$ are shown in Fig 2. The SIW antennas are fed by a microstrip-to-SIW transition in order to excite the dominant mode of the SIW antenna. The high loss tangent $(\tan \delta)$ of the paper substrate and the long length of the SIW antennas result in high loss in the nonradiation bands. The slight frequency shift for the resonant frequency of the $3.6 \mathrm{GHz}$ antenna is due to the bonding layers which have slightly higher dielectric constant than the paper substrate. The measurements match the simulation very well and the measured radiation patterns of the both antennas are presented in Fig 3.

\section{RETRO-DIRECTIVE VAN ATTA ARRAY}

In this section, a dual-band retro-directive Van Atta array prototype for chipless RFID tag and sensor applications is proposed using inkjet printing technology. The designed SIW antennas operating at $3.6 \mathrm{GHz}$ and $5.8 \mathrm{GHz}$ are interwoven in order to achieve retro-directive property. The antenna array which is operating at the higher frequency range is placed in the middle of the antenna array operating at the lower frequency band in order to minimize the loss from the Ushaped interconnects of the antenna array for the higher frequency band. Due to the fact that the length of the outer Uinterconnect is longer than the inner one, the antennas which are operating at high band frequency should be embedded between the antennas which are operating at low frequency.

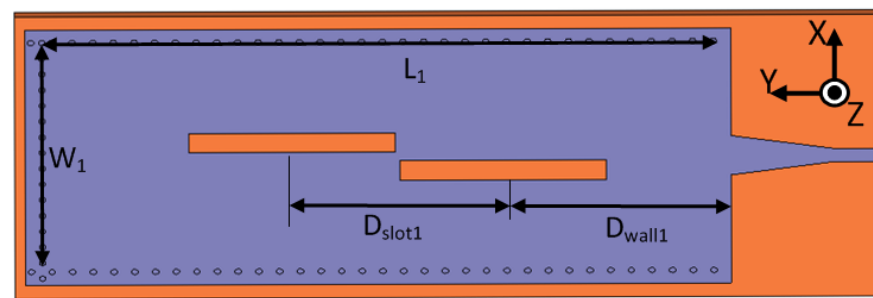

(a)

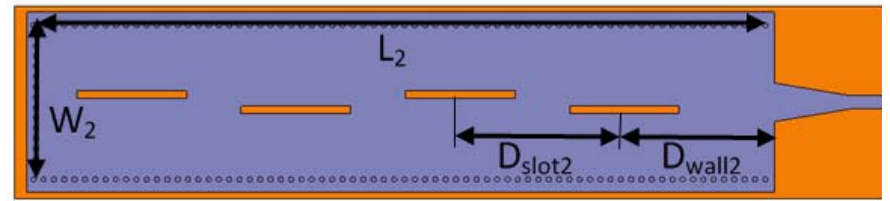

(b)

Fig 1. Geometry of SIW antennas on paper (a) $3.6 \mathrm{GHz}$. (b) $5.8 \mathrm{GHz}$ $\mathrm{W}_{1}=39 \mathrm{~mm}, \mathrm{~L}_{1}=89 \mathrm{~mm}, \mathrm{D}_{\text {slot } 1}=24.5 \mathrm{~mm}, \mathrm{D}_{\text {wall1 }}=24.5 \mathrm{~mm}, \mathrm{~W}_{2}=28 \mathrm{~mm}$, $\mathrm{L}_{2}=116 \mathrm{~mm}, \mathrm{D}_{\text {slot2 }}=25.5 \mathrm{~mm}, \mathrm{D}_{\text {wall } 2}=23.25 \mathrm{~mm}$
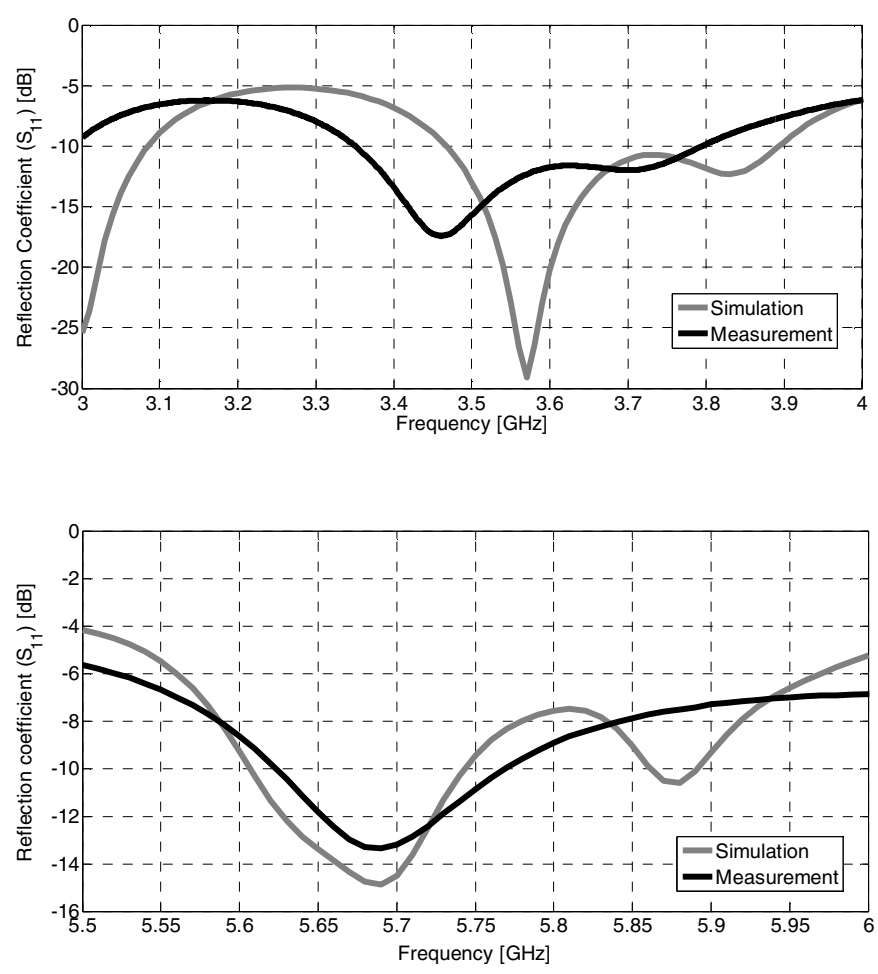

Fig 2. Reflection coefficients of antennas at (a) $3.6 \mathrm{GHz}$, (b) $5.8 \mathrm{GHz}$

Otherwise, the length of the interconnect for the higher frequency band is longer than the that for the lower frequency band which results in significant loss of the antenna array for the higher frequency band because of the long length of the interconnect in terms of wavelength. The designed reflector is inkjet-printed on paper substrate and it is shown in Fig 4. Each SIW element is sharing the via walls in order to reduce the size of the reflector as much as possible.

The designed retro-directive array is simulated in a bistatic environment in order to demonstrate its retro-directive 


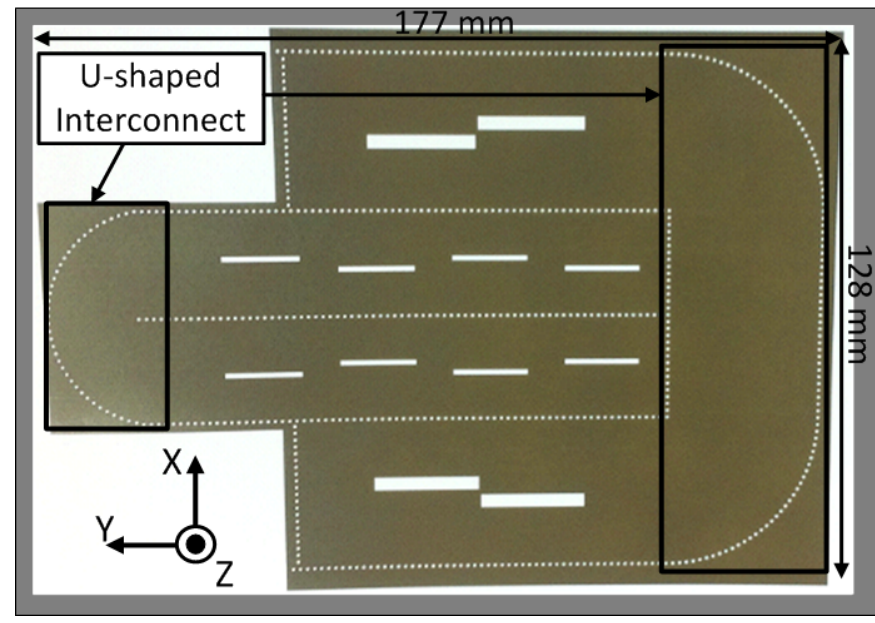

Fig. 4. Inkjet-printed dual-band Van Atta retro-directive reflector

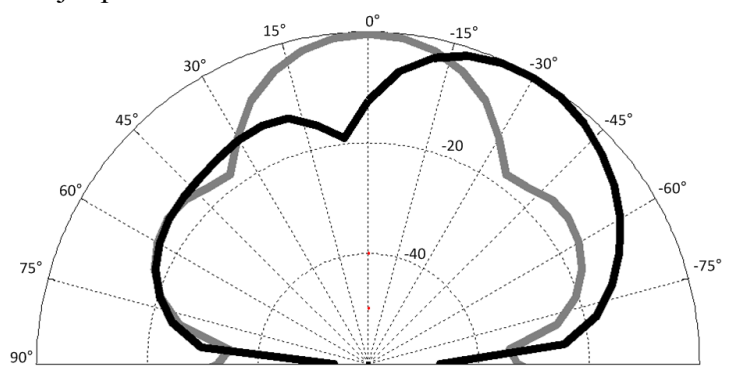

(a)

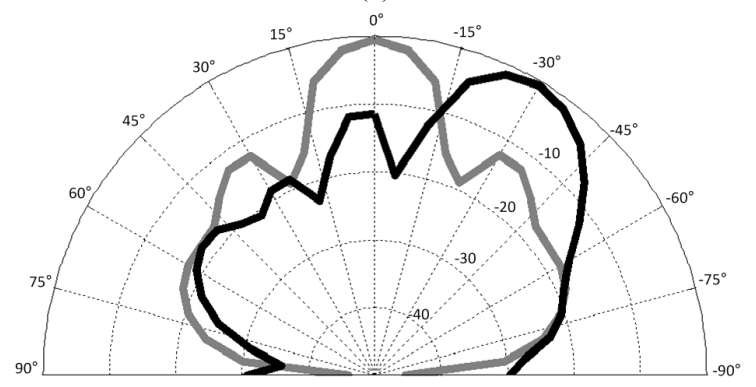

(b)

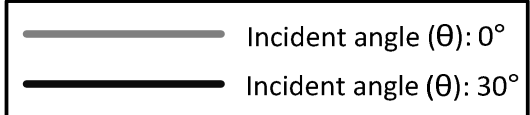

Fig. 5. Simulated bi-static scattered power pattern at (a) $3.6 \mathrm{GHz}$ (b) $5.8 \mathrm{GHz}$

property. The simulated normalized RCS patterns are shown in Fig 5 verifying the retro-directivity for incidence angles of 0 and 30 degrees. These RCS pattern verifies the retrodirectivity of the proposed design since the retro-directed power is detected. The low power level detection of $-15 \mathrm{dBm}$ is due to the low gain of the antennas due to the high-loss of paper substrate.

\section{SENSOR INTERROGATION BENCHMARKING}

As a proof-of-concept demonstration of the sensor interrogation capabilities of the proposed dual-band retro-

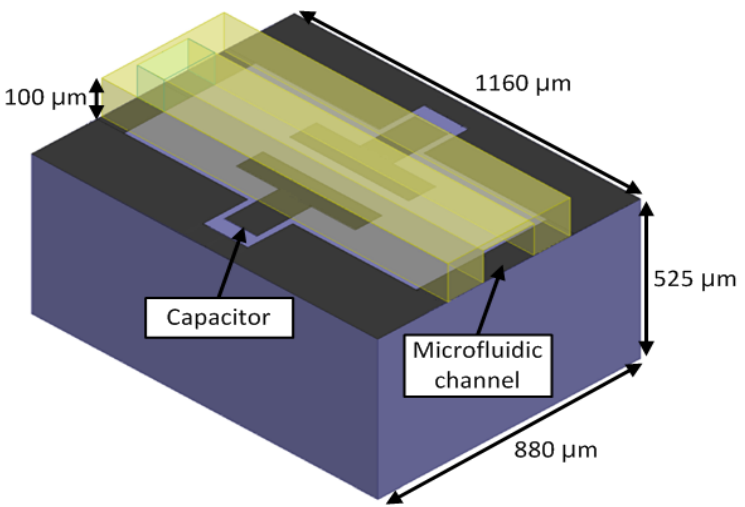

(a)

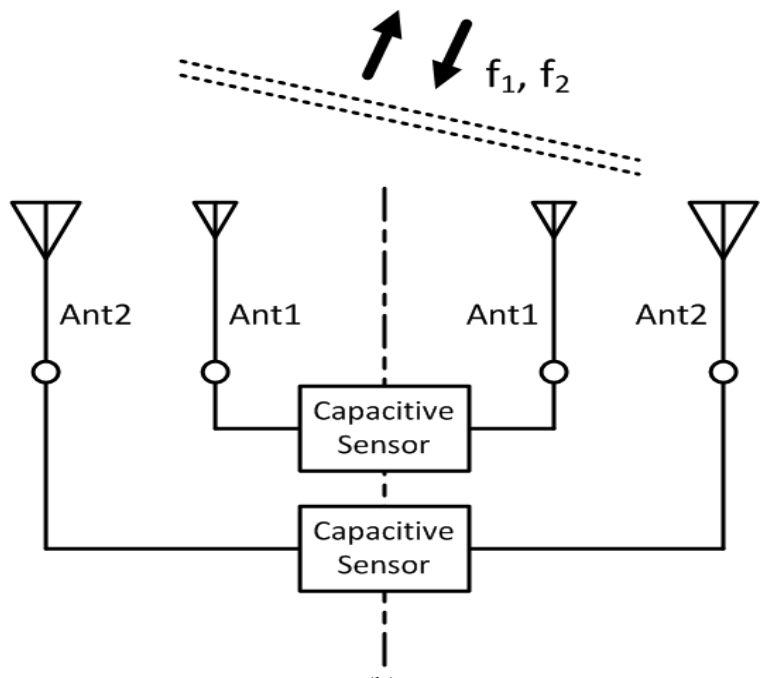

(b)

Fig. 6. (a) 3D schematic of the proposed micro-fluidic capacitive microsensor and (b) block diagram of dual-band retro-directive sensor

directive antenna, we are planning to test the RCS variability of the recently developed microfluidic capacitive microsensor shown in Fig 6(a) [10]. The microsensor is made up of a microfluidic channel that runs along a gap oriented parallel to a capacitor. The capacitance of the capacitor varies according as the fluid fills the gap of the capacitor. Results with horn antenna interrogation have demonstrated an RCS variability of $4 \mathrm{dBm}$ for the temperature range of $24 \sim 33^{\circ} \mathrm{C}$, but the sensitivity is also dependent on the angle of illumination. It is expected that the use of the proposed retro-directive structure will significantly enhance the readability for the majority of illumination angles and offer a two-frequency temperaturedependent RCS variability verification (Fig 6(b)).

\section{CONCLUSION}

In this paper, an inkjet-printed passive SIW dual-band retrodirective array on paper substrate for chipless RFID tag and sensor applications is presented for the first time. Design requirements for the dual band Van Atta array are discussed and the dual band retro-directive reflector has been designed 
and investigated. The proposed topology features numerous advantages compared to previously reported dual-band reflect arrays including compactness, versatility for multiband implementations, completely passive operation and significantly reduced electromagnetic interference due to feeding structures.

\section{ACKNOWLEDGE}

The work of S. Kim, B. Cook, J. Cooper and M. M. Tentzeris was supported by NSF, NEDO, and IFC/SRC. The work of A. Georgiadis was supported by EU Marie Curie FP7-

PEOPLE-2009-IAPP 251557 and by the Spanish Ministry of Economy and Competitiveness project TEC 2012-39143.

\section{REFERENCES}

[1] R.Y. Miyamoto, and T. Itoh, "Retrodirective arrays for wireless communications," IEEE Microwave Magazine, vol.3, no.1, pp.71-79, Mar 2002.

[2] N.C. Karmakar, Handbook of Smart Antennas for RFID Systems, Wiley 2010.

[3] R.Y. Miyamoto, Q. Yongxi, and T. Itoh, "An active integrated retrodirective transponder for remote information retrieval-ondemand," IEEE Transactions on Microwave Theory and Techniques, vol.49, no.9, pp.1658-1662, Sep 2001.

[4] A. Traille, S. Bouaziz, H. Aubert, P. Pons, and M. Tentzeris, "A Novel Wireless Passive Temperature Sensor Utilizing Microfluidic Principles in Millimeter-Wave Frequencies," in proc., 2011 IEEE Sensors, Limerick, Ireland, pp.524-525, 2011.

[5] J. Cespedes, F. Giuppi, A. Collado, and A. Georgiadis, "A Retrodirective UHF RFID Tag on Paper Substrate," 2012 IEEE RFID-TA, Nice, Nov. 5-7, 2012.

[6] A. Ali, H. El-Shaarawy, and H. Aubert, "Millimeter-Wave Substrate Integrated Waveguide Passive Van Atta Reflector Array," IEEE Transactions on Antennas and Propagation, in proc., 2012

[7] M. Bozzi, A. Georgiadis, and K. Wu, "Review of substrateintegrated waveguide circuits and antennas," IET Microwaves, Antennas \& Propagation, vol.5, no.8, pp.909-920, June 2011.

[8] D-J. Jung, J. N. Hansen, and K. Chang, "Dual-band/dualpolarized microstrip patch antenna for dual-band heterodyne retrodirective array," Microwave and Optical Technology Letters, Volume 54, Issue 12, pages 2781-2784, December 2012.

[9] G. S. Shiroma, R. Y. Miyamoto, and W. A. Shiroma, "FullDuplex Dual-Frequency Self-Steering Array Using Phase Detection and Phase Shifting," IEEE Transactions on Microwave Theory and Techniques, Vol. 54, No. 1, Jan. 2006

[10] S. Bouaziz, , F. Chebila, A. Traille P. Pons, H. Aubert, and M. M. Tentzeris, "Novel Micro-fluidic Structures for Wireless Passive Temperature Telemetry Medical Systems Using Radar Interrogation Techniques in Ka-band," in proc., IEEE Antennas and Wireless Propagation Letters, 2012 